\title{
Spatial distribution of spectral parameters of high latitude geomagnetic disturbances in the $\mathrm{Pc5} / \mathrm{Pi3}$ frequency range
}

\author{
N. V. Yagova ${ }^{1}$, V. A. Pilipenko ${ }^{1,2}$, L. N. Baransky ${ }^{1}$, and M. J. Engebretson ${ }^{3}$ \\ ${ }^{1}$ Institute of the Physics of the Earth, Moscow 123995, Russia \\ ${ }^{2}$ Space Research Institute, Moscow 117997, Russia \\ ${ }^{3}$ Augsburg College, Minneapolis, MN 55454, USA
}

Received: 5 May 2010 - Revised: 2 September 2010 - Accepted: 15 September 2010 - Published: 28 September 2010

\begin{abstract}
We analyze spectral parameters of the geomagnetic disturbances within the $1-4 \mathrm{mHz}(\mathrm{Pc} 5 / \mathrm{Pi} 3)$ frequency range for 29 observatories from polar to auroral latitudes. The main object of this study is the broadband (noise) background under quiet and moderately disturbed conditions. To obtain a quantitative description of background high-latitude long period ULF activity the log-log dependence of the spectral power on frequency is expanded over Legendre polynomials, and the coefficients of this expansion (spectral moments) are used to describe the most common features of these spectra. Not only the spectral power, but also the spectral slope and higher spectral moments, averaged over relatively long time intervals, demonstrate a systematic dependence on corrected geomagnetic (CGM) latitude, $\Phi$, and magnetic local time, MLT. The 2-D distributions of the spectral moments in $\Phi$-MLT coordinates are characterized by existence of structures, narrow in latitude and extended in MLT, which can be attributed to the projections of different magnetospheric domains. Spatio-temporal distributions of spectral power of elliptically (P-component) and randomly ( $\mathrm{N}$ component) polarized signal are similar, but not identical. The $\mathrm{N}$-component contribution to the total signal becomes non-negligible in regions with a high local activity, such as the auroral oval and dayside polar cusp. The spectral slope indicates a larger relative contribution of higher frequencies upon the latitude decrease, probably, as a result of the resonant effects in the ULF noise. The higher spectral moments are also controlled mostly by CGM latitude and MLT and are fundamentally different for the polarized and non-polarized components. This study is a step towards the construction of an empirical model of the ULF wave power in Earth's magnetosphere.
\end{abstract}

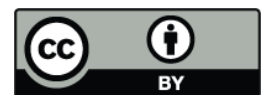

Correspondence to: N. V. Yagova (nyagova@yandex.ru)
Keywords. Magnetospheric physics (Auroral phenomena; Magnetopause, cusp, and boundary layers; MHD waves and instabilities)

\section{Introduction}

Ultra-low frequency (ULF) geomagnetic disturbances, monitored and recorded on the ground, are a manifestation of hydromagnetic (MHD) waves generated in the Earth's magnetosphere. The lower boundary of the ULF frequency range, about $1 \mathrm{mHz}$, is determined by the length of the last closed field line and the Alfvén velocity in the outer magnetosphere. The energy accumulated and transported by long period ULF waves is larger than that of any other electromagnetic wave processes in the near-Earth environment.

ULF activity comprises both narrowband $(\mathrm{Pc})$ and broadband $(\mathrm{Pi})$ pulsations. At high geomagnetic latitudes at frequencies of a few $\mathrm{mHz}$ the narrowband pulsations (Pc5-6) occur from time to time, while the broadband irregular variations (Pi3) are more ubiquitous. ULF geomagnetic activity has been continuously recorded for several decades at hundreds of ground stations and numerous satellites, but most studies have focused only on narrowband pulsations, while noise parameters have not been analyzed yet in a systematic way. Thus, a major part of the available information on high latitude ULF activity has been excluded from analysis, because the occurrence rate of narrowband pulsations is low as compared with that of broadband pulsations with the same amplitudes and in the same frequency bands.

At mid-latitudes the diurnal trends and seasonal variations of amplitudes of ULF noise and polarization in a wide frequency range from Pc2 to Pc5 $(0.002-0.1 \mathrm{~Hz})$ were found to be very similar at different stations with similar magnetic latitudes (Bloom and Singer, 1995). The amplitude and spectral

Published by Copernicus Publications on behalf of the European Geosciences Union. 
slope of Pc5 geomagnetic fluctuations at $L=4.4$, although correlated with global geomagnetic indexes, cannot be entirely characterized by them (Surkan and Lanzerotti, 1974). In the frequency range $10^{-4}-10^{-2} \mathrm{~Hz}$ the power spectrum of geomagnetic fluctuations at mid-latitudes can be approximated as $\propto f^{-3}$ (Lanzerotti et al., 1990).

At high latitudes, besides traditional Pc5 pulsations, specific polar cap Pi3 activity exists. The spectral power of high latitude Pi3 fluctuations is controlled by the extramagnetospheric factors (Yagova et al., 2004, 2007). Longterm observations at high latitudes established a connection between the interplanetary magnetic field (IMF) and ionospheric current systems, which resulted in several empiricalanalytical models of large-scale electromagnetic variations: IZMEM (Papitashvili et al., 1994), Weimer (Weimer, 2001), KRM (Kamide et al., 1981), AMIE (Richmond and Kamide, 1988), and field-aligned current model (Papitashvili et al., 2001), parameterized by the IMF direction/strength, season, and hemisphere. Statistical models of the spatial distribution of Pc4-5 wave power in the magnetosphere, derived from the data of highly-elliptical spacecraft were developed by Anderson et al. (1990) and Takahashi and Anderson (1992). However, so far there is no model to quantify the instantaneous ULF power distribution all over the globe. The elaboration of such a model may be a useful tool for insight into global short-scale electrodynamics and its quantitative description. Moreover, such model could be used for the prediction of magnetic field variability, necessary to estimate the hazard risk for satellite and ground technological systems (Perry et al., 2005).

This study is an attempt to lay a basis for a new parameterized numerical model of high latitude long period ULF activity, that utilizes high-precision maps of the spatial distribution of ULF spectral parameters. At this step we concentrate on the background daily variations of ULF spectral parameters, averaged over a relatively long time period. The analysis of ULF parameters during extreme space weather events will be the subject of our next study.

There have been numerous studies aimed on localization of the ionospheric projections of various magnetospheric domains and their boundaries by using pulsation properties observed on the ground. The location of maximal dayside Pc5 amplitude and linear polarization was supposed to indicate the position of the last closed field line (Samson, 1972). The narrow-band Pc5 pulsations at about $5 \mathrm{mHz}$ were found to be common below the last closed field line and random at higher latitudes (Lanzerotti et al., 1999).

The problem of cusp localization with the help of the analysis of pulsation parameters turned out to be more complicated, so the results of different research groups seem contradictory and strongly dependent on technique applied. At the expected dayside cusp latitudes the temporal variations of broadband ULF activity were found to dominate the spatially localized variations traditionally associated with the cusp position (Engebretson et al., 1995; Posch et al., 1999). On the other hand, Szuberla et al. (2000) have revealed with polarization analysis some spatial ULF features related to a possible cusp projection.

Intense broadband noise, Polar Boundary Intensifications (PBI), was suggested to be an indicator of the poleward auroral boundary in the midnight sector (Zesta et al., 2006). This activity is very localized. The examination of spectral coherence and time variations of spectral power at these latitudes has shown that ULF fluctuations may be uncoupled between geographically nearby areas, possibly projected into different magnetospheric regions (Yagova et al., 2004).

Impulsive or quasi-periodic variations of the solar wind dynamic pressure can cause Pc5-6 oscillations that are highly coherent throughout all the high-latitude region (Kim et al., 2002). A positive correlation between the amplitude of the geomagnetic pulsations on the ground and solar wind dynamic pressure oscillations in the Pc5/6 frequency range exists not only for high-amplitude events, but permanently at any amplitude of the pressure oscillations (Yagova et al., 2007). High latitude long-period pulsations can be driven also by quasi-periodic IMF oscillations (Prikryl et al., 1999; Pilipenko et al., 2000; Kepko et al., 2002). The Kelvin-Helmholtz instability of the magnetopause is one of the oldest mechanisms suggested to explain Pc5 morphological properties (Samson, 1972). The same mechanism of high-latitude Pc5 pulsations was suggested by Clauer et al. (1997), but at the ionospheric convection shear reversal boundary.

In ULF studies different techniques of automatic discrimination between noise and signal have been applied. Analysis of power spectral density was used for separation between narrow-band signals and broadband noise for auroral Pc5 (Posch et al., 2003; Baker et al., 2003), and high-latitude Pc3-4 (Ponomarenko et al., 2002; Chugunova et al., 2007). These methods enabled the authors to find a distinction in the behavior of narrowband signals and broadband noise. Polarization methods provide a tool to split the spectrum of recorded time series into elliptically polarized and randomly polarized parts. Their mathematical basis was developed in optics (Born and Wolf, 1964) and then successfully applied to ULF pulsation analysis (Fowler et al., 1967). In the polar cap the diurnal and seasonal variations of narrowband and broadband pulsations, as well as their inter-station spectral coherency, were found to be very similar (Yagova et al., 2002). However, in general, the possibility of different origins of long period ULF pulsations and noise with different polarization properties, has not been thoroughly examined.

In this paper we suggest a new approach to the statistical analysis of diurnal and 2-D spatial distributions of spectral characteristics of both broadband (Pi3) and narrowband (Pc5) pulsations. The structure of the paper is as follows: the observational data and data processing methods are described in Sect. 2, Sect. 3 summarizes the results of analysis, which are discussed in Sect. 4. 
Table 1. Stations and latitude zones.

\begin{tabular}{|c|c|c|c|c|c|c|c|c|}
\hline \multirow{3}{*}{$\begin{array}{l}\text { Boundaries } \\
\text { CGM Lat }\end{array}$} & \multirow{3}{*}{$\begin{array}{l}\text { Station } \\
\text { code }\end{array}$} & \multirow{3}{*}{$\begin{array}{l}\text { Network } \\
\text { code }\end{array}$} & \multicolumn{2}{|c|}{ Geographic } & \multicolumn{2}{|c|}{ CGM } & \multirow{2}{*}{\multicolumn{2}{|c|}{$\begin{array}{c}\text { MLT } \\
\text { midnight, }\end{array}$}} \\
\hline & & & Latitude & Longitude & Latitude & Longitude & & \\
\hline & & & & & & & UT & $\mathrm{LT}$ \\
\hline \multicolumn{9}{|l|}{$65-67$} \\
\hline & $\mathrm{ABK}$ & WDC-E & 68.4 & 18.8 & 65.2 & 102.7 & $21: 21$ & $22: 36$ \\
\hline & LRV & WDC-E & 64.2 & 338.3 & 65.3 & 68.1 & 00:05 & $22: 38$ \\
\hline & TIK & WDC-E & 71.6 & 128.8 & 65.8 & 197.1 & $16: 04$ & $00: 39$ \\
\hline & MAS & IMAGE & 69.5 & 23.7 & 66.1 & 106.9 & 21:06 & $22: 41$ \\
\hline & PBQ & WDC-E & 55.3 & 282.3 & 66.3 & 357.8 & 05:05 & $23: 54$ \\
\hline & TRO & WDC-E & 69.7 & 18.9 & 66.5 & 104.0 & $21: 16$ & $22: 32$ \\
\hline \multicolumn{9}{|l|}{$67-69$} \\
\hline & RBT & CARISMA & 58.2 & 256.3 & 67.2 & 317.8 & $07: 25$ & $00: 30$ \\
\hline & FSM & CARISMA & 60.0 & 248.1 & 67.6 & 305.5 & 08:10 & $00: 42$ \\
\hline \multicolumn{9}{|l|}{$69-71$} \\
\hline & FCC & WDC-E & 58.8 & 265.9 & 69.1 & 331.9 & $06: 35$ & $00: 19$ \\
\hline & YKC & WDC-E & 62.5 & 245.5 & 69.7 & 298.8 & $08: 35$ & $00: 57$ \\
\hline & KTN & CPPMN & 75.9 & 137.7 & 70.3 & 201.6 & $15: 48$ & 00:59 \\
\hline \multicolumn{9}{|l|}{$71-72$} \\
\hline & EKP & CARISMA & 61.1 & 265.9 & 71.3 & 331.5 & $06: 36$ & $00: 20$ \\
\hline & $\mathrm{BJN}$ & WDC-E & 74.5 & 19.2 & 71.4 & 108.7 & $20: 59$ & $22: 16$ \\
\hline \multicolumn{9}{|l|}{$72-73$} \\
\hline & HOP & IMAGE & 76.5 & 25.1 & 72.9 & 115.9 & $20: 32$ & $22: 12$ \\
\hline \multicolumn{9}{|l|}{$73-75$} \\
\hline & CNT & CARISMA & 65.8 & 248.8 & 73.3 & 302.1 & $08: 22$ & $00: 57$ \\
\hline & BLC & WDC-E & 64.3 & 264.0 & 74.0 & 327.1 & $06: 52$ & $00: 28$ \\
\hline & HRN & WDC-E & 77.0 & 15.6 & 74.06 & 110.4 & $20: 54$ & $21: 56$ \\
\hline \multicolumn{9}{|l|}{$75-77$} \\
\hline & LYR & IMAGE & 78.2 & 15.8 & 75.2 & 112.9 & $20: 45$ & $21: 48$ \\
\hline & GDH & WDC-E & 69.3 & 306.5 & 75.9 & 39.8 & $02: 24$ & $22: 50$ \\
\hline & NAL & IMAGE & 78.9 & 12.0 & 76.1 & 112.1 & $20: 49$ & $21: 37$ \\
\hline \multicolumn{9}{|l|}{$77-80$} \\
\hline & GHV & MACCS & 68.6 & 264.1 & 78.0 & 324.7 & $06: 59$ & $00: 35$ \\
\hline & TAL & CARISMA & 69.5 & 266.5 & 79.1 & 328.5 & $06: 46$ & $00: 32$ \\
\hline & IGL & MACCS & 69.3 & 278.2 & 79.2 & 352.5 & $05: 25$ & $23: 58$ \\
\hline & CLD & MACCS & 70.5 & 291.4 & 79.3 & 18.6 & $03: 52$ & $23: 18$ \\
\hline \multicolumn{9}{|l|}{$80-82$} \\
\hline & NRD & DMI & 81.6 & 343.3 & 81.0 & 105.7 & $21: 16$ & 20:09 \\
\hline \multicolumn{9}{|l|}{$82-84$} \\
\hline & RES & MACCS & 74.7 & 265.1 & 83.2 & 319.7 & $07: 16$ & $00: 56$ \\
\hline & SVS & DMI & 76.0 & 294.9 & 83.7 & 35.5 & $02: 46$ & $22: 26$ \\
\hline \multicolumn{9}{|l|}{$84-86$} \\
\hline & THL & DMI & 77.5 & 290.8 & 85.4 & 32.9 & $02: 57$ & $22: 20$ \\
\hline \multicolumn{9}{|l|}{$86-88$} \\
\hline & ALE & WDC-E & 82.5 & 297.7 & 87.0 & 101.9 & $21: 34$ & $17: 24$ \\
\hline
\end{tabular}




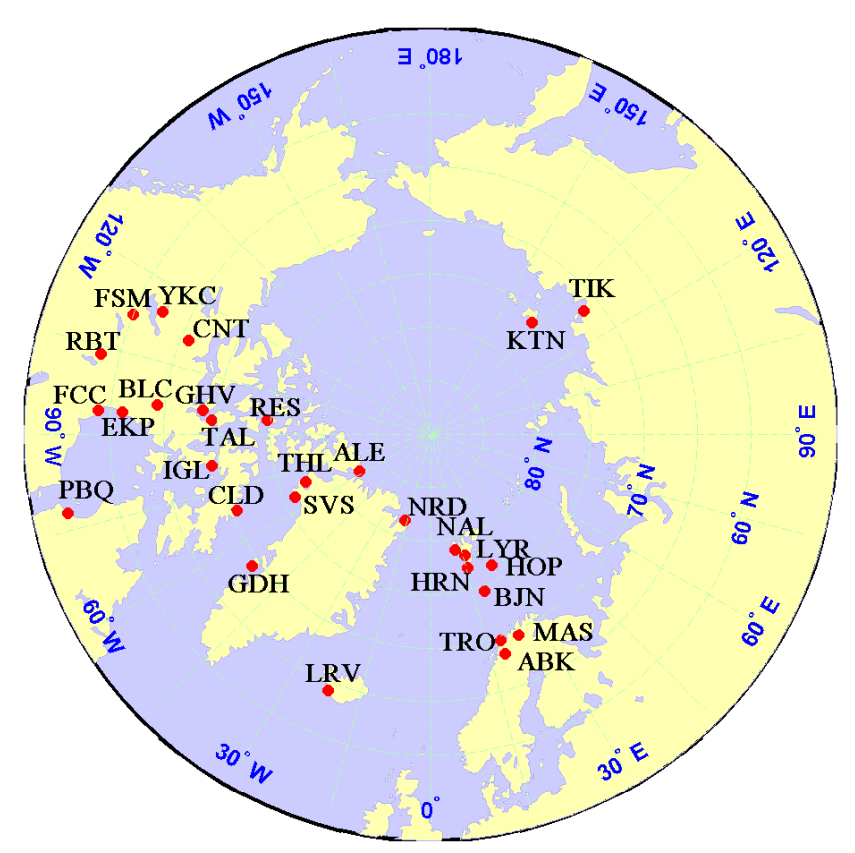

Fig. 1. A map with the station positions in geographic coordinates.

\section{Observational data and processing technique}

\subsection{Observational data}

For this study we use 1-min fluxgate magnetometer data from several magnetometer arrays: IMAGE (www.ava.fmi.fi/image/index.html), Greenland DMI magnetometer network (web.dmi.dk/projects/chain/), CARISMACANMOS (portal.cssdp.ca:8080/ssdp/jsp/dataProducts.jsp), MACCS (space.augsburg.edu/space/index.html), Circumpan Pacific Magnetometer Network (denji102.geo.kyushu-u. ac.jp/denji/obs/cpmn/cpmn_obs_e.html), and stations from the WDC for Geomagnetism, Edinburgh (www.wdc.bgs.ac. $\mathrm{uk} / \mathrm{catalog} / \mathrm{master} \cdot \mathrm{html})$. The stations selected are located from $65^{\circ}$ to $87^{\circ} \mathrm{CGM}$ latitudes $(\Phi)$ in all longitudinal sectors. Data from more than 50 stations with $\Phi>65^{\circ}$ were preanalyzed, and 29 of them with good data coverage have been chosen for detailed analysis (see map in Fig. 1). The coordinates of each station and the local time of MLT midnight are given in Table 1. Two groups of stations, located in relatively narrow intervals of geomagnetic longitudes in Scandinavia and Greenland $\left(100-110^{\circ} \mathrm{E}\right)$ and in Canada $\left(300-330^{\circ} \mathrm{E}\right)$, have station coverage from polar to auroral latitudes, which enable us to construct 2-D distributions of ULF parameters in $\Phi$-MLT coordinates. At other longitudes the coverage is more fragmented. In all important latitudinal zones, i.e. the polar cap, cusp, and auroral oval, including its poleward and equatorward boundaries, there are at least several stations at different longitudes. This enables us to analyze the latitudinally averaged parameters, take into account the influence of geographic latitude, and exclude the intervals with intensive artificial noise. We have chosen for analysis 23 months with the best data coverage from February 1997 through December 1998 .

\subsection{Data processing}

A routine data pre-processing procedure includes elimination of instrumental spikes, interpolation to a common sampling rate, and linear detrending. Intervals with long gaps are excluded from the analysis, and short data gaps (several points) are filled with interpolated values. We used a two-hour running time window for spectral estimates. A time window is considered valid for the analysis if it includes at least $90 \mathrm{~min}$ of continuous records. Thus, the actual length of time interval varies from 90 to $120 \mathrm{~min}$ and we do not use window overlapping to avoid any variations in the degree of overlapping.

Using the polarization analysis technique (Born and Wolf, 1964; Fowler et al., 1967) the horizontal signal on the ground is expanded into two components: an elliptically polarized component $(\mathrm{P})$ and a randomly polarized component $(\mathrm{N})$.

The following technique of quantitative analysis of ULF spectra $S(f)$ in the frequency range $\left(f_{\min }, f_{\max }\right)$ has been developed and applied. This technique is based on the analysis of the log-log spectrum, i.e. the dependence of the logarithm of spectral power $\sigma(F)=\log \left(S / S_{0}\right)\left(S_{0}=1 \mathrm{nT}^{2} / \mathrm{Hz}\right)$ on the normalized logarithm $F$ of frequency $f$

$F=\frac{2 F_{U}-\left(F_{\max }+F_{\min }\right)}{F_{\max }-F_{\min }}$

where $F_{U}=\log (f), F_{\max }=\log \left(f_{\max }\right), \quad F_{\min }=\log \left(f_{\min }\right)$. This log-log spectrum is expanded into Legendre polynomials

$\sigma(F) \simeq \sigma_{N}(F)=\sum_{n=0}^{N} L_{n} \overline{P_{n}}(F)$

Here $\overline{P_{n}}$ is the n-th normalized Legendre polynomial (Korn and Korn, 1968)

$\overline{P_{n}}(x)=\sqrt{\frac{2 n+1}{2}} P_{n}(x), \quad P_{n}(x)=\frac{1}{2^{n} n !} \frac{d^{n}}{d x^{n}}\left(x^{2}-1\right)^{n}$

$\left(P_{0}=1, P_{1}=x, P_{2}=\frac{3}{2} x^{2}-\frac{1}{2}, P_{3}=\frac{5}{2} x^{3}-\frac{3}{2} x, \ldots\right)$ and $L_{n}$ is an expansion coefficient. The coefficient $L_{0}$ is proportional to the averaged over spectral domain logarithm of the spectral power, $L_{0}=\sqrt{2} \bar{\sigma}$.

A straight line in the log-log presentation corresponds to a power-law dependence of power spectral density on frequency $S(f) \propto f^{-\alpha}$ (colored noise). The coefficient $L_{1}$ is proportional to the spectral slope index $\alpha$

$L_{1}=-k_{1} \alpha, \quad k_{1}=\frac{\left(F_{\max }-F_{\min }\right)}{\sqrt{6}}$

The spectral slope index $\alpha$ together with the spectral power $L_{0}$ give information that is sufficient for rough estimates of 
typical Pi3 spectra. In order to keep the same units as in our previous paper (Yagova et al., 2007), instead of the coefficients $L_{0}$ and $L_{1}$ we use further the parameter $\sigma_{0}$ equal to the value of the linear approximation of the log-log spectral power at lowest boundary of the frequency interval, and the spectral slope $\alpha$.

The next spectral moment, $L_{2}$, characterizes a form of power spectrum. A spectrum with a maximum (minimum) in the center of the frequency band corresponds to negative (positive) $L_{2}$ values, respectively. The central frequency

$f_{\mathrm{c}}=\sqrt{f_{\min } f_{\max }}$,

determined from $f_{\mathrm{c}}=\log \left(f_{\mathrm{c}}\right)=\left(F_{\min }+F_{\max }\right) / 2$, and for the frequency interval analyzed $(1<f<4 \mathrm{mHz}) f_{\mathrm{c}}=2 \mathrm{mHz}$. Further we use the parameter $Q=-L_{2}$ to get a natural presentation with positive values for a narrowband (high Qfactor) signal. Hence, $Q>0$ corresponds to a spectrum with a maximum at the central frequency, while a spectra with maxima at the ends of the frequency band correspond to negative $Q$. Absolute values of $Q$ indicates deviation of the $\log$-log spectrum from straight line (colored noise), for the colored-noise spectrum $Q=0$.

Higher coefficients $L_{n}(n>2)$ correspond to a spectrum with several maxima. Even coefficients describe symmetric modes with the number of spectral minima/maxima for positive/negative values equal to $n / 2$, and odd coefficients describe antisymmetric spectral distributions.

An illustration of this technique is given in Fig. 2, which shows the dependence of the logarithm of the spectral power $\sigma(F)$ on the normalized logarithm of the frequency (black solid line) and its approximations (Eq. 2). In this example the function $\sigma_{2}$ with two non-zero coefficients is sufficient for a rough estimate, and $\sigma_{4}$ gives a rather accurate approximation, which is not improved significantly when higher-order terms are included.

The behavior of pulsations' spectral parameters may be different at different timescales. Diurnal variations and latitude distribution of the spectral power are known to vary essentially from day to day in dependence on season (Ballatore et al., 1998) and solar wind parameters (Engebretson et al., 1998). However, the $\Phi$-MLT distributions of spectral power at timescales of about several months demonstrate some features, common for different time intervals. In order to study the $\Phi$-MLT dependencies of various spectral parameters, the whole region of observations has been divided into several latitudinal zones, and the two-year observational period has been divided into four half-year sub-intervals. The boundaries of these latitudinal zones and the station coordinates, sorted over CGM latitude, are given in Table 1. The spectral parameters are calculated for each half-year time interval and for each value of $\Phi$ and MLT. ULF spectral parameters for different sub-intervals and sites with close CGM latitudes are compared to estimate qualitatively the reproducibility of the pulsation "portrait".

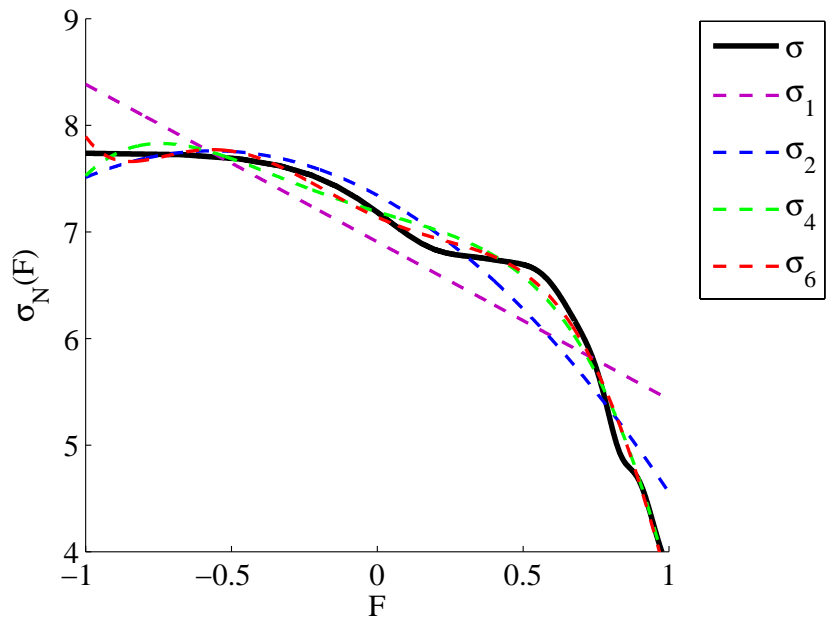

Fig. 2. An example of $\sigma(F)$ dependence and its approximation $\sigma_{N}(F)$ with Eq. (2). The experimental curve is shown with a solid black line, and the fitting curves are given with dashed lines. The approximation orders are given with at the legend.

Diurnal variations of each coefficient $L_{n}$ in Eq. (2) can be approximated by harmonic functions as follows

$L_{n}=\sum_{k=0}^{K} C_{n k} \cos \left(\frac{2 \pi}{T} k t\right)+\sum_{k=1}^{K} S_{n k} \sin \left(\frac{2 \pi}{T} k t\right)$

Here $C_{n 0}$ is the daily averaged value of $L_{n}$, and $T=24 \mathrm{~h}$. Coefficients $C_{n 1}$ and $S_{n 1}$ describe the first harmonic with one daily maximum. The positive (negative) first cosine harmonic $C_{n 1}$ at zero $S_{n 1}$ corresponds to a midnight (noon) maximum. If the first sine harmonic $S_{n 1} \neq 0$ and $C_{n 1}=0$ the maximum is located at the dawn-dusk meridian (06-18 UT), and a morning maximum corresponds to $S_{n 1}>0$. The parameter $K$ in Eq. (6) is the number of Fourier coefficients sufficient for the description of diurnal variation. It will be shown in the next section that for all the spectral moments $L_{n}$ the averaged diurnal variation is rather accurately described by Eq. (6) with $K=2$.

In the description of the spatial distribution of ULF spectral parameters the relative position with respect to auroral boundaries is important. Although both the spatial distribution and boundaries vary with time, the average picture is relatively stable and corresponds to a quiet or moderately disturbed magnetosphere. All the results have been calculated twice: for the total period of observations and for days with low and moderate activity (days of magnetic storms with Dst $<-100 \mathrm{nT}$ and 7 days after, i.e. the storm recovery phase, are excluded). The results have been almost identical, so only the results averaged over the entire observational period are shown further. The negligible contribution of stormtime intervals to the general pattern is due to a small number of storms during the analyzed period. However, storm-time ULF spatial and MLT distributions may differ dramatically from those during quiet times and from one storm to another 

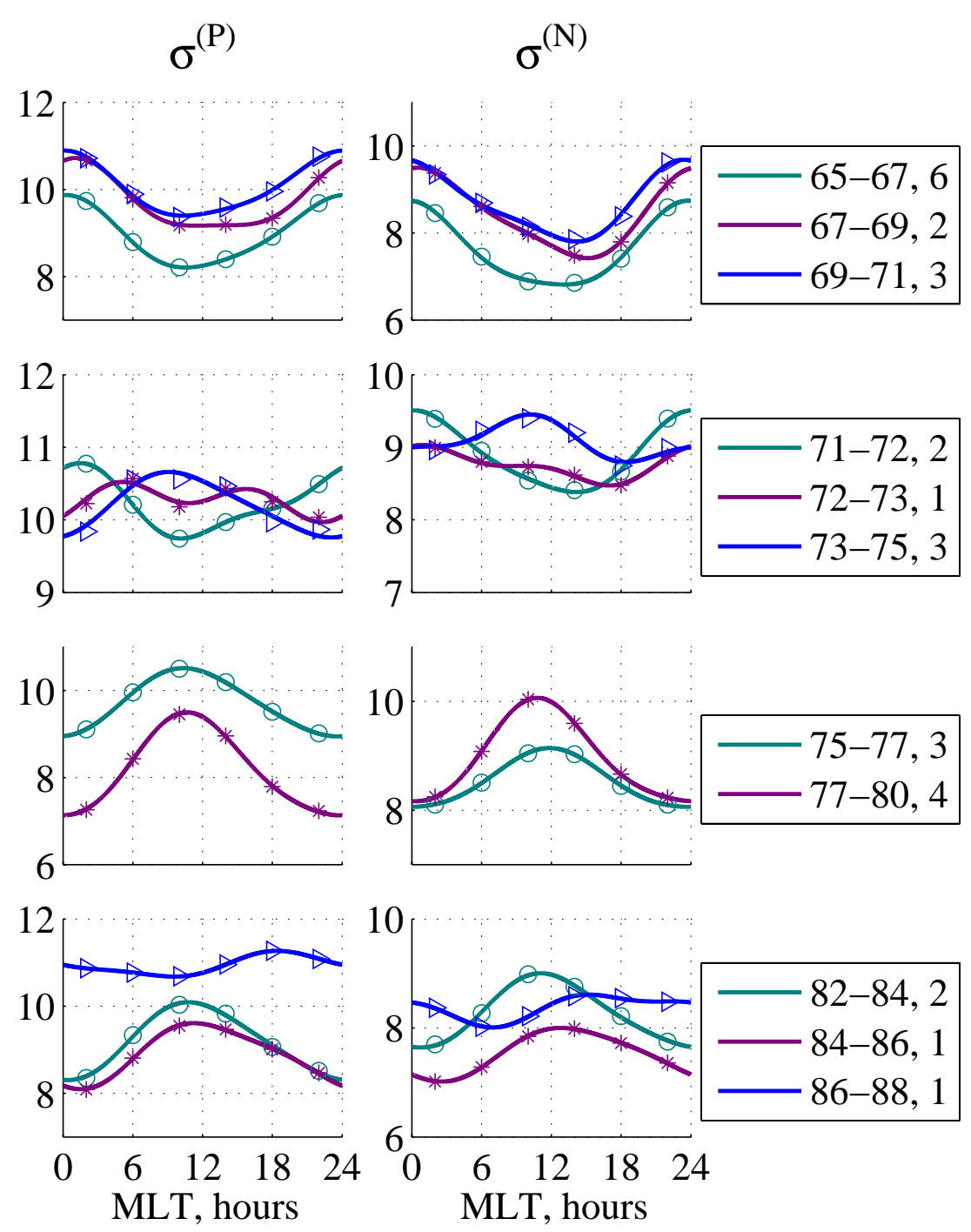

Fig. 3. Diurnal variations of the logarithm of the spectral power $\sigma$, averaged over CGM latitude $\Phi$, and their approximations according to Eq. (6) at $K=2$. Observed values are given with markers, and lines show the results of approximation. Boundaries of zones and number of stations are given at the legend.

(Kleimenova and Kozyreva, 2005; Lee et al., 2007; Pilipenko et al., 2010).

\section{Results of the analysis: spatial distribution of Pi3 spectral parameters}

\subsection{Spectral power}

The diurnal variations of ULF spectral power at latitudinallyaveraged zones, given in Table 1, are shown in Fig. 3 for P-component (left) and N-component (right). The values of $\sigma$ for each MLT interval are given with markers and its approximation by the Fourier harmonics are shown with solid lines. For all the latitudes the dependence $\sigma(t)$ is approximated with the Eq. (6) at $K=2$, so the coefficients of the approximation can be used for a compact presentation of the spectral parameters.

The shape of diurnal variation changes systematically with latitude from a typical auroral type with one maximum near MLT midnight (upper panel) to the cusp-like type with a near-noon maximum (3-rd panel). Between those latitudes a transitional type with several maxima (2-nd panel) is observed. Diurnal variations of polarized and non-polarized components are approximately similar, but for all latitudes the spectral power of the $\mathrm{P}$-component is several times higher than that of the $\mathrm{N}$-component. The $\mathrm{P} / \mathrm{N}$ ratio is maximal at the lowest and the highest latitudes, i.e. below the equatorial boundary of the auroral oval and deep in the polar cap, while near the center of the auroral zone and the nominal cusp position the randomly polarized power becomes comparable with the elliptically polarized power. 

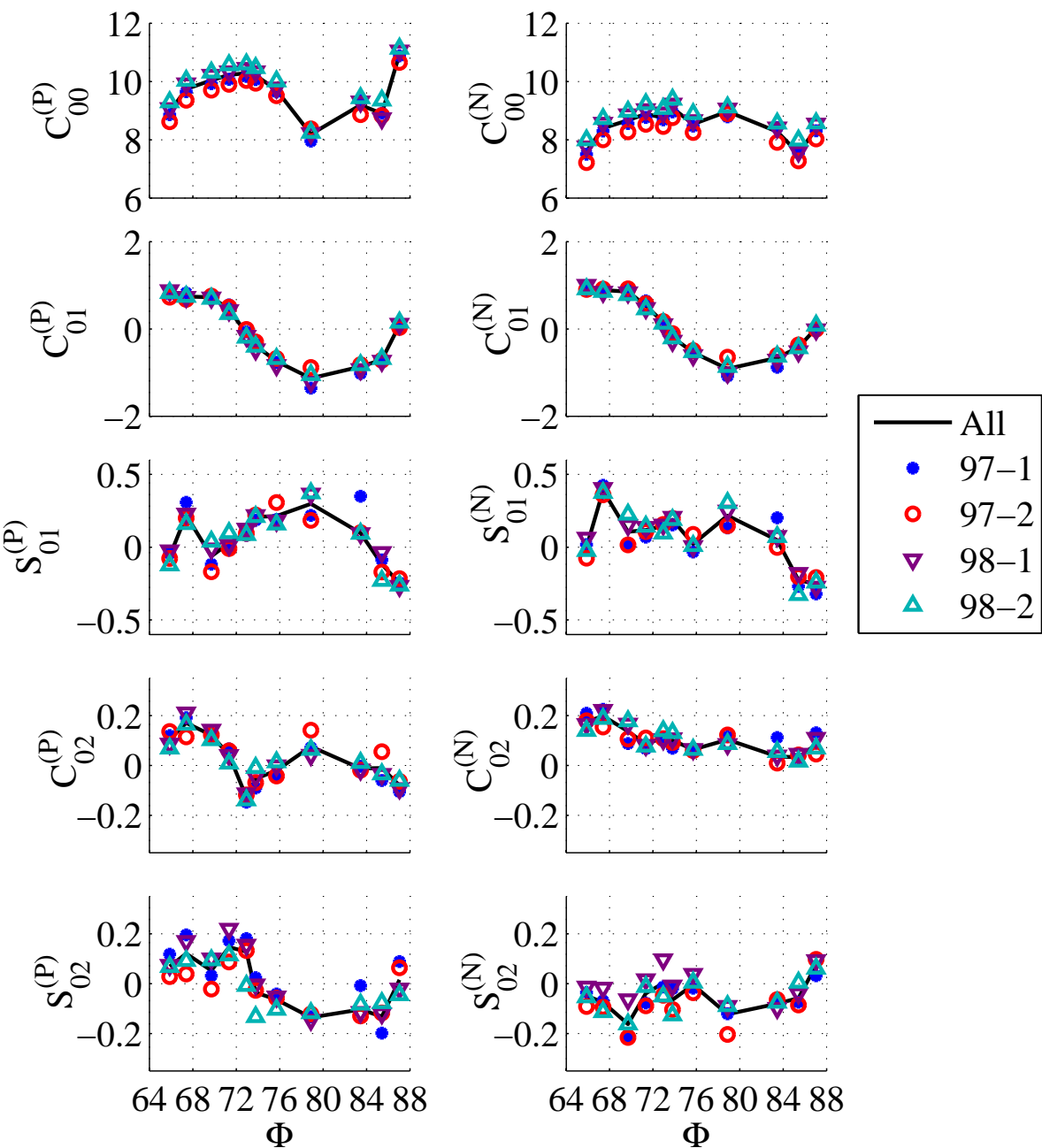

Fig. 4. Coefficients of the Fourier expansion (6) of $\sigma$. The $\mathrm{P}$ and $\mathrm{N}$ components are given in the left and right panels, respectively. The results for the whole two-year observational interval are shown by a solid line, and the results for half-year intervals are shown by markers. The years are given in first two numbers on the legend, and the third number corresponds to half-year intervals: 1 is January-June, and 2 is July-December.

For the stations located deep in the polar caps, the difference in diurnal variations becomes significant even at small geographical separations. Therefore, at the bottom panel of Fig. 3 individual patterns are given for three polar cap stations $\operatorname{NRD}\left(\Phi=81^{\circ}\right)$, THL $\left(85.4^{\circ}\right)$, and $\operatorname{ALE}\left(87^{\circ}\right)$. While the diurnal variation at THL is still a cusp-like one with a single maximum near noon, at the polarmost station ALE the diurnal variation almost vanishes. This result agrees with observations in the Southern Hemisphere along the $80^{\circ}$ magnetic meridian showing that the spectral power is not controlled totally by $\Phi$ and MLT, but depends significantly also on longitude Yagova et al. (2004). At the station in the Northern Hemisphere with nearly the same CGM latitude, NRD $\left(\Phi=81^{\circ}, \lambda=106^{\circ}\right)$, the maximum of $\mathrm{Pi} 3$ spectral power is shifted to afternoon hours. A similar picture was found by Yagova et al. (2004) in the Southern Hemisphere at SBA $\left(\Phi=-80^{\circ}, \lambda=327^{\circ}\right)$, located at nearly the same geographic latitude as NRD, but in the opposite MLT sector. For both NRD and SBA the difference between the solar (LT) and magnetic (MLT) local times is about $4-5 \mathrm{~h}$. The LT-MLT difference at the polarmost station ALE is $6.5 \mathrm{~h}$ and there a wide evening maximum of spectral power is also seen in $\mathrm{P}$ component. For all other high-latitude stations in the Northern Hemisphere the LT-MLT difference does not exceed $1.5 \mathrm{~h}$ and the diurnal variation of spectral power demonstrate only a near-noon maximum. We exclude the station NRD from the further analysis, because for it the difference between magnetic and local time is big and the results, obtained from NRD data cannot be correctly extrapolated for all the $\Phi=80^{\circ}$ latitude zone. Contrary, data from ALE is included, because it is located very near the CGM North pole, the circle $\Phi=87^{\circ}$ is small, and for any point there the MLT-LT difference is high. Hence, the diurnal variation at ALE is representative for all the locations at the same $\Phi$. 

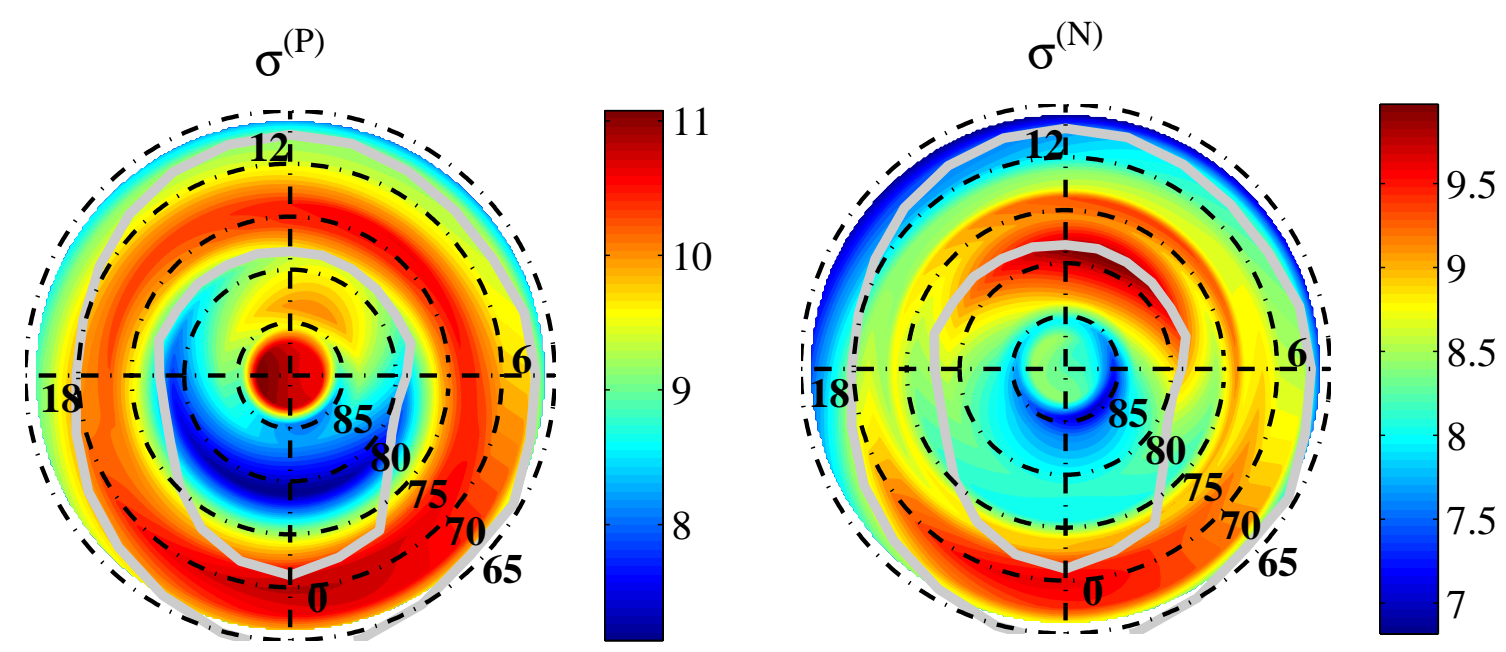

Fig. 5. 2-D distribution of $\sigma$ calculated according to Eq. (6) with $K=2$ in $\Phi$-MLT coordinates for P-component (left) and N-component (right). Two-year averaged positions of auroral boundaries are shown with white thick grey lines.
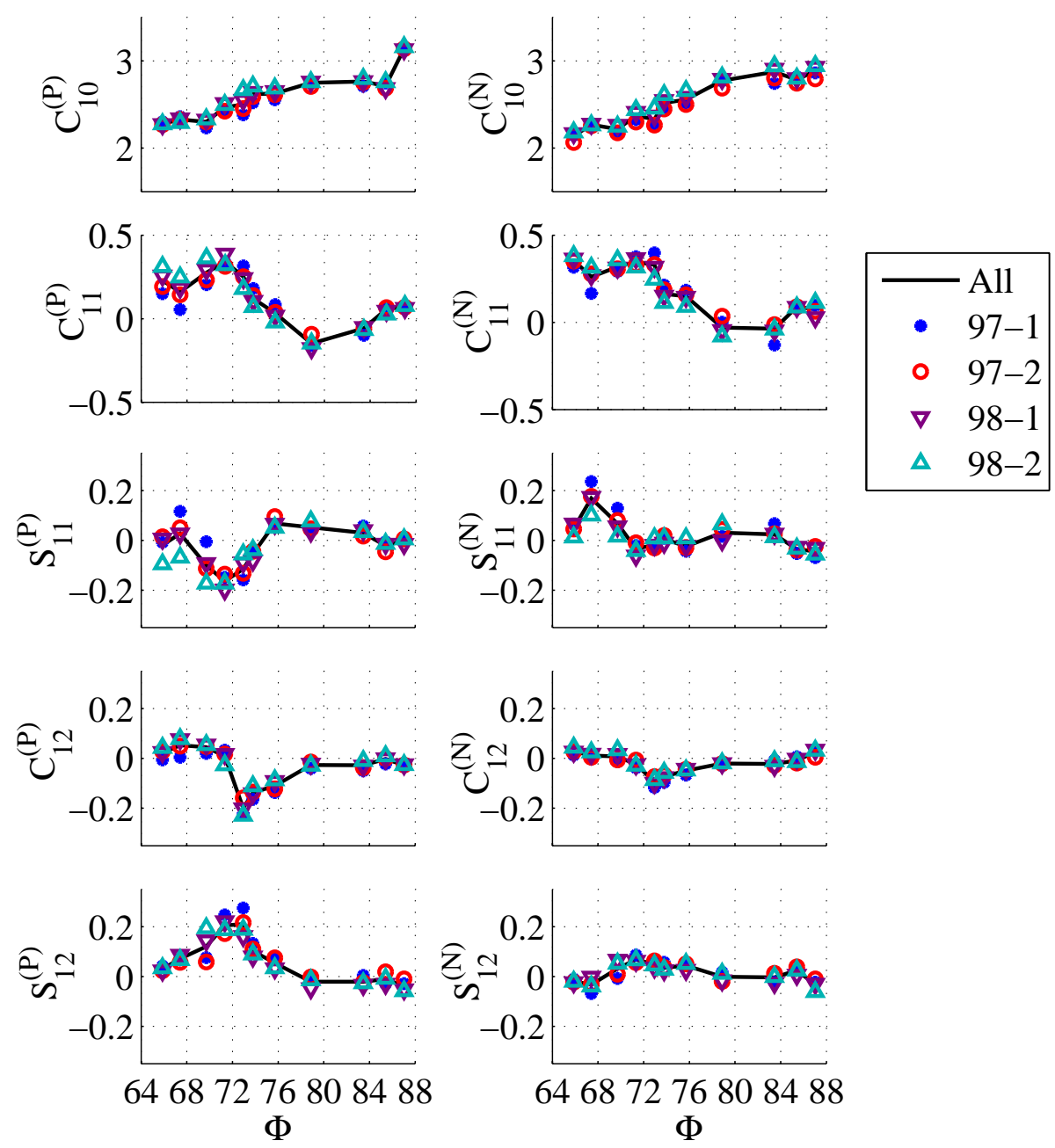

Fig. 6. Coefficients of the Fourier expansion (6) of the spectral slope $\alpha$ for the P-component (left) and the N-component (right) in the same format as in Fig. 4. 

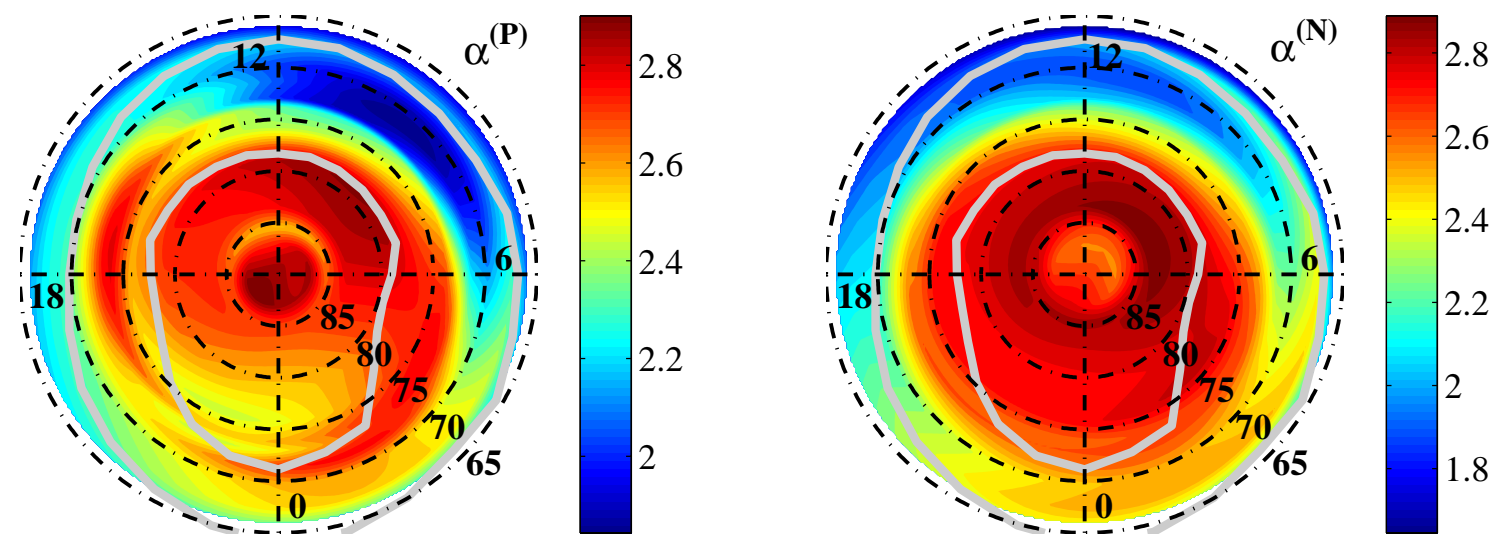

Fig. 7. 2-D distribution of the spectral slope $\alpha$ in $\Phi$-MLT coordinates in the same format as in Fig. 5. The warm colors (large spectral slopes) correspond to spectra dominated by low frequencies and cool colors (low spectral slope) indicate that the spectra are enriched with high frequencies.

The consistency of diurnal variations has been checked by dividing the whole two-year interval into sub-intervals. The coefficients of the Fourier expansion (Eq. 6) for four halfyear intervals (markers) and the whole period of observations (solid lines) are given in Fig. 4. The zero coefficient $C_{00}$ (daily averaged value of $\sigma$ ) is reproduced well for both components in all latitudinal zones. It is substantially higher for the P-component than for the $\mathrm{N}$-component except in the zone of high local ULF activity near $\Phi=78^{\circ}$. Here the daily averaged non-polarized power is even higher than the polarized power. The maximum of $\sigma$ deep in the polar cap is seen only in the P-component.

The first cosine harmonic, $C_{01}$, also demonstrates a high reproducibility for all the half-year intervals and a qualitatively similar latitude dependence for both components. The switch from positive to negative values of $C_{01}$ at $\Phi \approx 73^{\circ}$ indicates the transition from an auroral-like to a cusp-like type of diurnal variation. The coefficient $S_{01}$ is small in comparison with $C_{01}$, which means that the daily main maximum is located near the noon-midnight line. The reproducibility of $S_{01}$ is also high, except in the $82^{\circ}<\Phi<85^{\circ}$ zone for the P-component (third left-hand panel). The absolute values of the coefficients corresponding to the half-day harmonics are smaller than elder coefficients and the reproducibility is worse. However, the zones of positive/negative values of the coefficients for both components can be seen from the bottom panels of Fig. 4 . The coefficient $C_{02} \geq 0$ for the $\mathrm{N}$-component at all latitudes, indicating an occurrence of morning maximum, while for the P-component there are two regions with an evening maximum $\left(C_{02}<0\right)$ at auroral $\left(\Phi=73^{\circ}\right)$ and polar $\left(\Phi=87^{\circ}\right)$ latitudes. The sign of $S_{02}$ is opposite for the $\mathrm{P}$ and the $\mathrm{N}$ component at $\Phi<70^{\circ}$. At higher latitudes the latitude dependence of the $S_{02}$ coefficient is similar for both components, changing from negative to positive deep in the polar cap.
Summarizing, we can say that the average diurnal variation of long-period ULF spectral power at all the stations incluses into the analysis can be compactly presented with five parameters only (the daily average value and two harmonics with corresponding amplitude and phase). In the next paragraphs the same technique will be applied to higher spectral moments.

Figure 5 shows the 2-D distribution of the average logarithm of the spectral power in $\Phi$-MLT coordinates for both $\mathrm{P}$ and $\mathrm{N}$ components. Boundaries of the auroral oval in the Figs. 5, 7, and 9 are calculated with the OVATION technique (sd-www.jhuapl.edu/Aurora/ovation/) and averaged over the whole two-year period of observations. The following structures are clearly seen in both $\mathrm{P}$ and $\mathrm{N}$ components:

- the nighttime maximum at $67^{\circ}<\Phi<70^{\circ}$;

- the morning maximum at $73^{\circ}<\Phi<75^{\circ}$ (the red arc between 06:00 and 12:00 MLT in the left-hand panel and the yellow arc in the right-hand panel);

- the deep nighttime minimum at $82<\Phi<85^{\circ}$.

Contrary, the near-noon enhancement at $77^{\circ}<\Phi<80^{\circ}$ is mostly evident in the $\mathrm{N}$-component and the near-polar maximum of $\sigma$ is seen in the P-component only.

Hence, a polarized signal dominates almost everywhere, but the contribution of a randomly polarized signal becomes significant in the auroral oval and dayside polar cusp. The $\Phi$ MLT maps with polarized/non-polarized power distributions can be used as an additional tool for search of ULF discriminators of the ionospheric projection of the magnetospheric domains.

\subsection{Spectral slope}

Similarly to the spectral power, the spectral slope $\alpha$ demonstrates a regular diurnal variation with one or two daily 

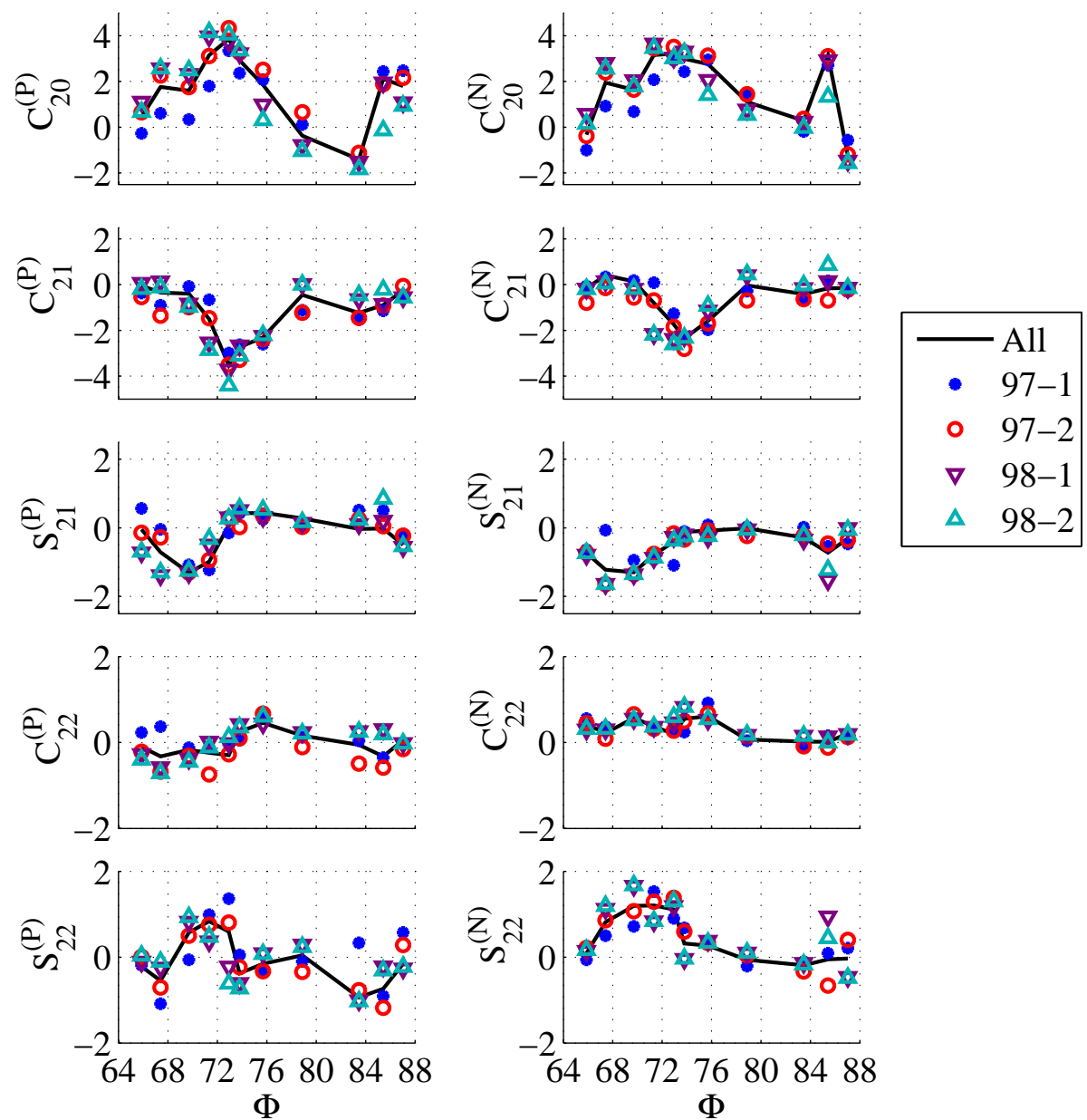

Fig. 8. Coefficients of the Fourier expansion (6) of the parameter $Q$ for the P-component (left) and the N-component (right) in the same format as in Fig. 4.

maxima at all latitudes. This dependence can also be approximated with good accuracy with a Fourier approximation (Eq. 6) at $K=2$. The latitude dependencies for zoneaveraged Fourier coefficients are given in Fig. 6. The daily averaged spectral slopes for both components (upper panels) are well reproduced for all the time sub-intervals. The latitudinal variations of these slopes are very similar for both $\mathrm{P}$ and $\mathrm{N}$ components.

Only near the geomagnetic pole, higher values of the spectral slope are seen for the P-component. Thus, the polar maximum of the polarized signal (Figs. 4,5 ) is mostly due to the ULF activity at the lowest frequencies of the interval analyzed $(f \approx 1 \mathrm{mHz}$ ).

The non-zero Fourier coefficients describing harmonics of the spectral slope diurnal variation have a good reproducibility in time and display a clear difference between two components. For the P-component at $\Phi<68^{\circ} C_{11}^{(\mathrm{P})}>0$ and $S_{11}^{(\mathrm{P})} \simeq 0$, indicating an occurrence of a nighttime maximum. At higher latitudes $\left(68^{\circ}<\Phi<75^{\circ}\right)$ a negative sine harmonic occurs, therefore the maximum of the spectral slope shifts to the dusk sector. Near the nominal cusp latitude $\left(77^{\circ}<\Phi<80^{\circ}\right) C_{11}^{(\mathrm{P})}<0$ whereas $S_{11}^{(\mathrm{P})} \simeq 0$, therefore the spectral slope has a near-noon maximum. At polar cap latitudes all the coefficients go to zero and the diurnal variation vanishes. For the $\mathrm{N}$-component the first cosine harmonic is nonnegative at all latitudes, i.e. a night-time maximum dominates. The difference between two components is maximal for the half-day harmonic coefficients $C_{12}$ and $S_{12}$ at $\Phi<75^{\circ}$ : a non-negligible half-day harmonic exists at these latitudes only for the P-component.

2-D distributions of spectral slopes for both components are shown in Fig. 7. For both components a daytime minimum of $\alpha$ exists at $69^{\circ}<\Phi<72^{\circ}$, but for the polarized signal it is concentrated at pre-noon and morning hours, whereas for the non-polarized component it covers a wider range of MLT from dawn to dusk. The morning minimum of $\alpha^{(\mathrm{P})}$ is located near the well-known region of morning Pc5 activity. However, not only classical Pc5 pulsations contribute to this effect, but also broadband disturbances at the same frequencies, as evident from $\alpha^{(\mathrm{N})}$ distribution. A zone 

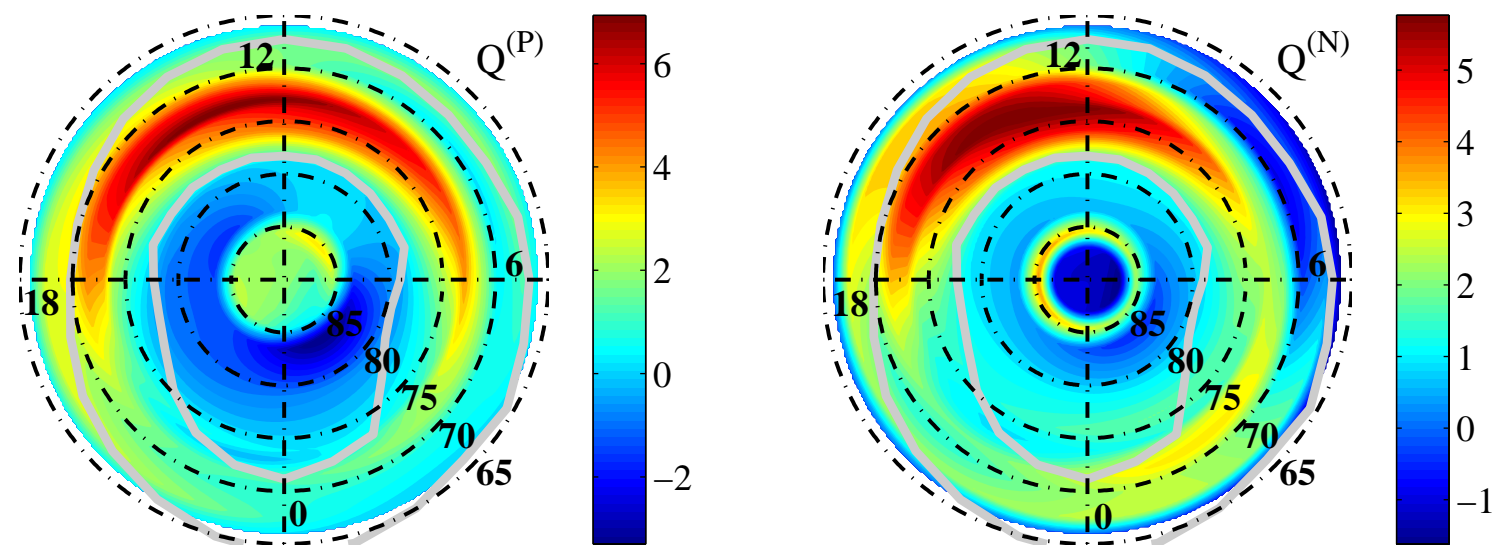

Fig. 9. 2-D distribution of the parameter $Q$ in $\Phi$-MLT coordinates for the P-component (left) and the N-component (right) in the same format as in Fig. 5.

of high spectral slopes of the P-component exists at $73^{\circ}<$ $\Phi<76^{\circ}$. It has two maxima, at evening and pre-morning hours, centered near the line 04:00-16:00 MLT. These maxima for the majority of time intervals are inside the auroral oval, and closer to its poleward boundary. At $77^{\circ}<\Phi<80^{\circ}$, i.e. at nominal cusp latitudes, a daytime maximum of $\alpha$ can be seen. Finally, $\alpha^{(\mathrm{P})}$ has a maximum deep in the polar cap, in the region co-located with the polar maximum of spectral P-power (Fig. 5).

Summarizing the above results, we should highlight the high reproducibility of the spectral slope distribution. In general, $\alpha$ grows with latitude and becomes higher during the nighttime. In addition, several zones of maximal and minimal $\alpha$ exist at specific $\Phi$ and MLT. These zone are narrow in latitude $\left(2-3^{\circ}\right)$ and wide in MLT (up to $12-16 \mathrm{~h}$ ). The distribution of spectral slopes only partly coincides with the distribution of spectral power.

\subsection{Higher spectral moments}

The parameter $Q$, proportional to the third coefficient of the expansion (2) describes deviation of the spectrum from the color-noise and high positive $Q$ correspond to narrow-band signal at the central frequency $f_{\mathrm{c}}$ determined with Eq. (5). Similar to the spectral amplitude and slope, the average diurnal variations of $Q$ have one or two daily maxima and can be well approximated with the Eq. (6) at $K=2$.

The latitude dependencies of the Fourier coefficients are given in Fig. 8 in the same format as Figs. 4 and 6. The main features of the latitude dependence $C_{20}(\Phi)$ are well reproduced for both components; only the behavior of $C_{20}$ at auroral latitudes for one half-year interval (1997-1) differs from the other three sub-intervals. Daily averaged values of $Q\left(\propto C_{20}\right.$, upper panels) are maximal at latitudes $72^{\circ}<\Phi<73^{\circ}$. At higher latitudes, $\Phi>77^{\circ}, C_{20}^{(\mathrm{P})}$ decreases rapidly, whereas the latitude decrease of $C_{20}^{(\mathrm{N})}$ is slower. The first cosine harmonic coefficient $C_{21}$ for both components is negative in the center of the auroral zone and is almost zero beyond it. Absolute values of the coefficient $S_{21}$ are smaller, but their reproducibility is still good for both components almost at all latitudes. For the half day harmonic at low latitude segment of the profile $\left(\Phi<72^{\circ}\right)$ we find $C_{22}^{(\mathrm{P})}<0<C_{22}^{(\mathrm{N})}$. This corresponds to dominance of the flank narrowband activity for polarized disturbances, and noon-midnight activity for non-polarized ones. A zone of positive $C_{22}$ exists at $\Phi \approx 76^{\circ}$ for both components, and at higher latitudes the second cosine harmonic tends to zero for both components, i.e. narrowband flank activity disappears. Zone of non-negligible positive $S_{22}$, centered at $\Phi \approx 71^{\circ}$ exists in both components. Taking into account that near its maximum $\left|C_{22}\right| \ll\left|S_{22}\right|$, in this zone the maximum of narrowband activity should be centered at the line 03:00-15:00 MLT. Negative $S_{22}$ exists at $\Phi \approx 84^{\circ}$, i.e. deep in the polar cap in the P-component, again at nearly zero $C_{22}$. This indicates the occurrence of a signal with the spectra essentially different from color noise near the line 09:00-21:00 MLT.

2-D distributions of $Q$ in $\Phi$-MLT coordinates are shown in Fig. 9. The main structures, seen in both components, are the following:

- the dusk zone of moderate positive $Q$ at latitudes below $70^{\circ}$;

- the post-noon maximum at $72^{\circ}<\Phi<75^{\circ}$;

- two deep minima with $Q<0$ at afternoon and postmidnight hours in the polar cap $\left(82<\Phi<85^{\circ}\right)$.

The difference between two components can be seen both in the magnitudes and qualitative behavior of $Q$. Its maximal value $Q_{\max } \approx 7$ for the P-component and $Q_{\max } \approx 5$ for the Ncomponent. This means that spectra of elliptically polarized signals are more narrow-band in comparison with randomly polarized signals. The daytime (MLT $\approx 14)$ maximum of $Q$ at $72^{\circ}<\Phi<75^{\circ}$ is higher for the P-component, but while 


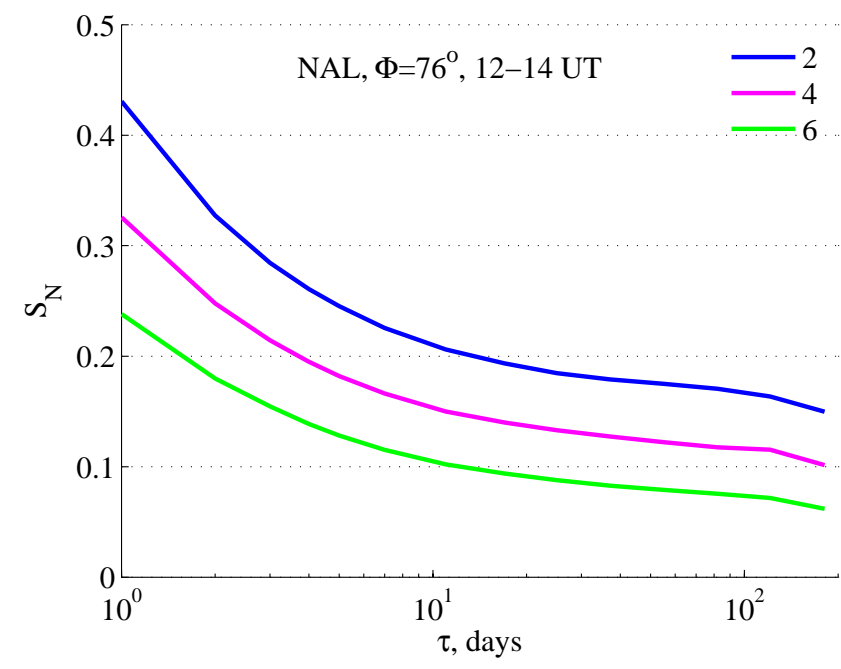

Fig. 10. Dependence of variance $S_{N}$ of the approximation (2) of $\bar{\sigma}(F, \tau), N=2,4,6$ on time of averaging $\tau$ for NAL station at afternoon time. Orders of the approximation are given with numbers at the legend.

$Q^{(\mathrm{P})}$ decreases monotonously with the azimuthal distance, $Q^{(\mathrm{N})}$ has an additional pre-dawn maximum. The distributions of $Q$ at latitudes $\Phi<80^{\circ}$ are similar for both components, while at higher latitudes the patterns do not coincide even qualitatively. A zone of essentially negative $Q^{(\mathrm{N})}$ exists deep in the polar cap, while $Q^{(\mathrm{P})}$ values are moderately positive there.

Hence, the second spectral moment $Q$ also shows relatively stable structures in $\Phi$-MLT coordinates. Some of them coincide with the known maxima of ULF activity, as morning and evening auroral Pc5 maxima, but others merit further study.

\subsection{Accuracy of the approximation at different timescales}

We analyze quantitatively the background properties of $\mathrm{Pc5} / \mathrm{Pi} 3$ pulsations at timescales from six months to two years approximating the pulsations' power spectrum (in loglog scale) with few first Legendre polynomials. The question arises about the accuracy of this approximation at different timescales and orders of the approximation. We denote as $\bar{\sigma}(F, \tau)$ the logarithm of spectral power $\sigma(F)$, averaged over time interval $\tau$, and as $\bar{\sigma}_{N}(F, \tau)$ its $\mathrm{N}$-th order approximation with Eq. (2). Here, to study diurnal variation of pulsations' spectra, we use $\tau$ in days, taking all the spectra for the same MLT intervals. The dispersion of this approximation is

$S_{N}^{2}(\tau)=\left\langle\left(\bar{\sigma}(F, \tau)-\bar{\sigma}_{N}(F, \tau)\right)^{2}\right\rangle$,

where $\langle\ldots\rangle$ means the averaging over normalized logarithm of frequency $F$. An example of the dependence of variance $S_{N}$ on the averaging time $\tau$ for three values of $N$ at one station and MLT interval is given in Fig. 10. The approximation at $N=2$ has accuracy better than $20 \%$ at $\tau>20$ days and it decreases to $10 \%$ for $N=6$ (note that we use natural logarithm of spectral power and the absolute values of $S$ correspond to relative error of the approximation of the spectral power at $S \ll 1$ ). 2-D distribution of the variance $S_{2}$ in $\Phi$-MLT coordinates is given in Fig. 11. It is calculated for seven stations located nearly along 110 magnetic meridian at $\tau=180$ days for P-component. The variance for $\mathrm{N}$-component (not shown) is lower for all the latitudes and local times. The dependence of variance on the order of approximation is similar at all latitudes and local times to that shown in Fig. 10, and using $N=4$ leads to almost twofold decrease of the variance. Thus for the major part of $\Phi$-MLT area the error of the approximation (2) at $N=2$ is less or about $10 \%$, and even maximal $25 \%$ error in Fig. 11 is small in comparison with the magnitudes of all the structures, seen in Figs. 5, 7 and 9. This means that the approximation (2) at $N=2$ is valid for the statistical estimates at timescale from several months to several years.

\section{Discussion and conclusions}

The newly introduced spectral moments, i.e. the spectral power, spectral slope $\alpha$, and parameter $Q$, to characterize the ULF noise demonstrate a systematic dependence on geomagnetic latitude and MLT. This gives the possibility of a quantitative description of the main features of ULF activity at high latitudes with a very limited number of parameters. For example, 15 coefficients $(3$ spectral $\times 5$ temporal) are sufficient for a rough description of diurnal variations of the ULF spectrum at any given latitude.

The spatial distributions and diurnal variations of spectral moments are not identical for elliptically (P-component) and randomly (N-component) polarized signals, and different types of ULF activity can be revealed better in one or another component. For example, the polar cap maximum of spectral power and spectral slope is seen only in the Pcomponent. In general, elliptically polarized signals dominate almost everywhere, while randomly polarized signals contribute most significantly to the total ULF power in the auroral oval and dayside polar cusp.

Rather surprisingly, the reproducibility of the $\Phi$-MLT distribution of the spectral slope $\alpha$ for different stations at close geomagnetic latitudes and different longitudes is even better than for the distribution of spectral power. Some zones of extreme $\alpha$ coincide with 2-D structures in spectral power and with expected projections of magnetospheric boundary layers. On the other hand, several zones of extreme values of $\alpha$ have been found which could not be associated with any specific magnetospheric domain. The high reproducibility of positions of red and blue spots in $\alpha$ maps (Fig. 8) should be further examined to find the responsible physical reasons. 


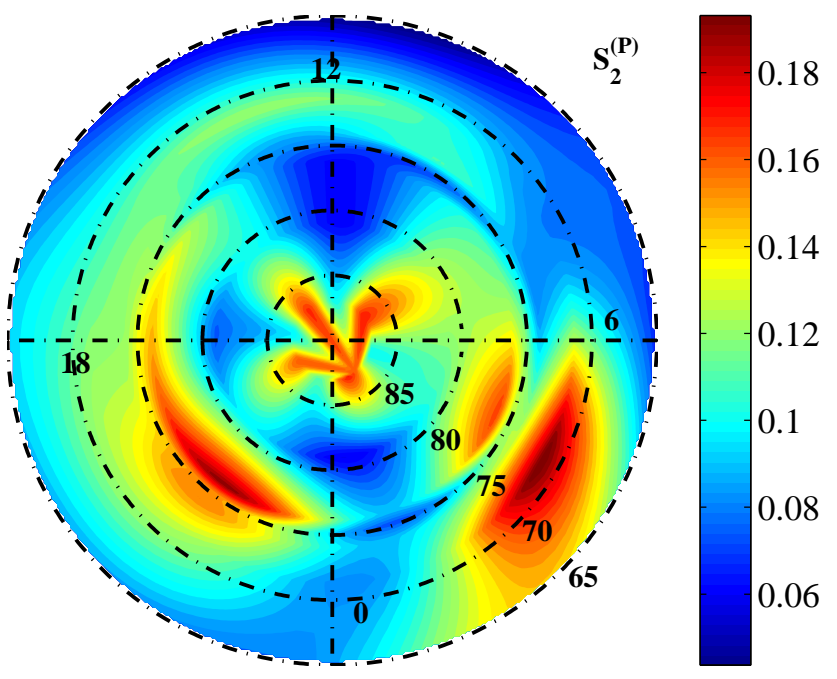

Fig. 11. Variance of the approximation (2) of $\bar{\sigma}(F, \tau)$ at $\tau=180$ days, $N=2$, calculated for P-component for seven stations, located along MM110 (ABK, BJN, HRN, LYR, NAL, NRD and ALE, station coordinates are given in Table 1) in dependence on CGM latitude and MLT.

The approximation of the spectrum with Eq. (2) at $N=2$ is accurate enough for statistical analysis. The reproducibility of the second-order spectral moment $Q$ for different time intervals and the existence of stable structures in its $\Phi$-MLT distribution shows that quantitative empirical modeling of high latitude ULF "noise" is feasible, at least in the Pc5/Pi3 frequency range.

We now formulate some approaches to the interpretation of the observed patterns. Elliptically polarized pulsations are generated by a source with large spatial scale, while randomly polarized signals could be the result of the superposition of local sources with random phases. The spectral power distribution of two components (Fig. 5) shows indeed that power of the $\mathrm{N}$-component becomes noticeable in zones of high local ULF activity, such as the center of the auroral zone and the nominal cusp position. As expected, spectra of the $\mathrm{N}$-component are more broadband than $\mathrm{P}$-component spectra, as can be seen from the comparison of the $Q$ parameter for the two components (Fig. 9). However, there are regions with opposite relation of polarization and spectral shape, where $Q^{(\mathrm{N})}>Q^{(\mathrm{P})}$, which can be explained by the existence of multiple local sources with band-limited spectra, but independent phases.

The 2-D distribution of the spectral slope in $\Phi$-MLT coordinates reveals the systematic growth of $\alpha$ with magnetic latitude and from day to night hours. Thus, the spectral slope increases with the increase of the expected Alfvén period. This regularity in the $\alpha(\Phi)$ behavior evidences the contribution of the Alfvén field line resonance into broadband ULF disturbances (see the most complete theory of field line resonance in the book Alperovich and Fedorov (2007)). The fun- damental frequencies of Alfvén field line resonance at the periphery of the Earth's magnetosphere, $\sim 1 \mathrm{mHz}$, fall into the frequency range considered in the present study.

Despite a very low quality of high latitude oscillations, ULF noise is controlled considerably by the same factors as regular Pc5 pulsations. The low quality of high-latitude ULF pulsations is not necessarily caused by dissipation, it can be the result of fluctuations of the system parameters (Coult et al., 2007). In addition to the regular growth of $\alpha$ with expected Alfvén period, the 2-D distribution of the spectral slope reveals some anomalous zones with extreme values of $\alpha$, e.g. near-noon region at $\Phi \approx 80^{\circ}$, i.e. in the proximity of the nominal cusp.

A possible correspondence of the spatial distribution of Pc5/Pi3 power with the magnetospheric field aligned currents was found in Potemra (1995) and Martines-Bedenko et al. (2003), but no statistical analysis of such correspondence has been made yet. Although this paper is not especially aimed at this problem, it follows from the above consideration (see Figs. 5, 7, 9) that the distribution of any spectral parameter does not match the ionospheric projection of the known current systems (Iijima and Potemra, 1976; Friis-Christensen et al., 1985). Moreover, the dependence of the spatial distribution of the ULF spectra on frequency and polarization (which follows from the non-monotonic distribution of $\alpha$ and $Q$ ) evidences that the spatial patterns are formed due to wave processes, but not just fluctuations of the global current systems.

The spatially-correlated day-to-day variations of $\mathrm{Pi} 3 \mathrm{spec}-$ tral power at high latitudes were explained by their dependence on extra-magnetospheric parameters (Yagova et al., 2007). A preliminary analysis of the inter-relations between the same group of extra-magnetospheric parameters and spectral slope revealed no dependence between them (Yagova et al., 2008). We suppose that the variations of spectral slope and higher spectral parameters are controlled mostly by the intra-magnetospheric factors and is a manifestation of an active response of the Earth's magnetosphere to the external forcing.

Acknowledgements. We thank for the provision of CPMN magnetometer data - K. Yumoto, Greenland - J. Watermann, and CARISMA-CANMOS - I. Mann. We thank the institutes who maintain the IMAGE Magnetometer Array. This study is supported by grant 07-05-00185 from the Russian Fund for Basic Research. The MACCS array and work at Augsburg College was supported by US National Science Foundation grant ATM-0827903.

Topical Editor R. Nakamura thanks K. Takahashi and F. Plaschke for their help in evaluating this paper.

\section{References}

Alperovich, L. S. and Fedorov, E. N.: Hydromagnetic Waves in the Magnetosphere and the Ionosphere, Series: Astrophysics and Space Science Library, vol. 353, 2009, XXIV, 418p., Hardcover, ISBN: 978-1-4020-6636-8, 2007. 
Anderson, B. J., Engebretson, M. J., Rounds, S. P., Zanetti, L. J., and Potemra, T. A.: A statistical study of Pc 3-5 pulsations observed by the AMPTE/CCE magnetic field experiment, 1, Occurrence distributions, J. Geophys. Res., 95, 10495-10523, 1990.

Baker, G. J., Donovan, E. F., and Jackel, B. J.: A comprehensive survey of auroral latitude Pc5 pulsation characteristics, J. Geophys. Res., 108(A10), 1384, doi:1029/2002JA009801, 2003.

Ballatore, P., Lanzerotti, L. J., and Maclennan, C. G.: Multistation measurements of Pc5 geomagnetic power amplitudes at high latitudes, J. Geophys. Res., 103, 29455-29465, 1998.

Born, M. and Wolf, E.: Principles of optics, Pergamon Press, London-New York-Paris, 1964.

Bloom, R. M. and Singer, H. J.: Diurnal trends in geomagnetic noise power in the Pc 2 through Pc 5 bands at low geomagnetic latitudes, J. Geophys. Res., 100, 14943-14953, 1995.

Chugunova, O., Pilipenko, V., Engebretson, M., and Rodger, A.: Statistical relationships between Pc3-4 occurrence at high latitudes in Antarctica and solar wind/IMF parameters, Geomagnetism Aeronomy, 46, 68-77, 2007.

Clauer, C. R., Ridley, A. J., Sitar R. J., Singer H. J., Rodger, A. S., Friis-Christensen, E., and Papitashvili, V. O.: Field line resonant pulsations associated with a strong dayside ionospheric shear convection flow reversal, J. Geophys. Res., 102, 45854596, 1997.

Coult N., Pilipenko, V., and Engebretson, M.: Suppression of resonant field line oscillations by a turbulent background, Planet. Space Sci., 55, 694-700, 2007.

Engebretson, M. J., Glassmeier, K.-H., Stellmacher, M., Hughes, W. J., and Luhr, H.: The dependence of high-latitude PcS wave power on solar wind velocity and on the phase of high-speed solar wind streams, J. Geophys. Res., 103, 26271-26283, 1998.

Engebretson, M. J., Hughes, W. J., Alford, J. L., Zesta, E., Cahill Jr., L. J., Arnoldy, R. L., and Reeves, G. D.: Magnetometer array for cusp and cleft studies observations of the spatial extent of broadband ULF magnetic pulsations of cusp/cleft latitudes, J. Geophys. Res., 100, 19371-19386, 1995.

Fowler R. A., Kotick, B. J., and Elliot, R. D.: Polarization analysis of natural and artificially induced geomagnetic micropulsations, J. Geophys. Res., 72, 2871-2883, 1967.

Friis-Christensen, E., Kamide, Y., Richmond, A., and Matsushita, S.: Interplanetary magnetic filed control of high-latitude electric fields and currents determined from Greenland magnetometer data, J. Geophys. Res., 91, 1325-1338, 1985.

Iijima, T. and Potemra, T. A.: The amplitude distribution of fieldaligned currents at northern high latitudes observed by TRIAD, J. Geophys. Res., 81, 2165-2174, 1976.

Kamide, Y., Richmond, A., and Matsushita, S.: Estimation of ionospheric electric fields, ionospheric currents, and field-aligned currents from ground magnetic records, J. Geophys. Res., 86, 801-813, 1981 .

Kepko, L., Spence, H. E., and Singer, H. J.: ULF waves in the solar wind as direct drivers of magnetospheric pulsations, Geophys. Res. Lett., 29, 1197, doi:10.1029/2001GL014405, 2002.

Kim, K.-H., Cattell, C.A., Lee, D.-H. , Takahashi, K., Yumoto, K., Shiokawa, K., Mozer, F. S., and Andre, M.: Magnetospheric responses to sudden and quasiperiodical solar wind variations, J. Geophys. Res., 107, 1406, doi:10.1029/2002JA009342, 2002.

Kleimenova, N. G. and Kozyreva, O. V.: Spatial-Temporal Dynamics of Pi3 and Pc5 Geomagnetic Pulsations during the Extreme
Magnetic Storms in October 2003, Geomagnetism and Aeronomy, 45, 71-79, 2005.

Korn, G. A. and Korn, T. M.: Mathematical handbook for scientists and engineers, McGraw-Hill Book Company, New York, San Francisco-Toronto-London-Sydney, 1968.

Lanzerotti, L. J., Maclennan, C. G., and Fraser-Smith, A. C.: Background magnetic spectra from $\sim 10^{-5}$ to $\sim 10^{5} \mathrm{~Hz}$, Geophys. Res. Lett., 17, 1593-1596, 1990.

Lanzerotti, L. J., Shono, A., Fukunishi, H., and Maclennan, C. G.: Long-period hydromagnetic waves at very high geomagnetic latitudes, J. Geophys. Res., 104, 28423-28435, 1999.

Lee, E. A., Mann, I. R., Loto'aniu, T. M., and Dent Z. C.: Global Pc5 pulsations observed at unusually low L during the great magnetic storm of 24 March 1991, J. Geophys. Res., 112, A05208, doi:10.1029/2006JA011872, 2007.

Martines-Bedenko, V. A., Pilipenko, V. A., Papitashvili, V. O., Engebretson, M. J., Watermann, J. F., and Newell, P. T.: Correspondence between ULF activity, field-aligned currents, and DMSP-based dayside magnetospheric domains, Geomagnetism and Aeronomy International, 4, 141-151, 2003.

Papitashvili, V., Belov, B., Faermark, D., Feldstein, Y., Golyshev, S., Gromova, L., and Levitin, A.: Electric Potential Patterns in the Northern and Southern Polar Regions Parameterized by the Interplanetary Magnetic Field, J. Geophys. Res., 99, 1325113262, 1994.

Papitashvili, V., Christiansen, F., and Neubert, T.: Field-aligned currents during IMF<0, Geophys. Res. Lett., 28, 3055-3058, 2001.

Perry, K. L., Hudson, M. K., and Elkington, S. R.: Incorporating spectral characteristics of Pc5 waves into three-dimensional radiation belt modeling and the diffusion of relativistic electrons, J. Geophys. Res., 110, A03215, doi:10.1029/2004JA010760, 2005.

Pilipenko, V. A., Fedorov, E. N., Engebretson, M. J., Papitashvili, V. O., and Watermann, J.: Poleward progressing quasi-periodic disturbances at cusp latitudes: The role of wave processes, J. Geophys. Res., 105, 27569-27588, 2000.

Pilipenko V., Kozyreva, O., Belakhovsky, V., Engebretson, M. J., and Samsonov, S.: Generation of magnetic and particle ??5 pulsations at the recovery phase of strong magnetic storms, Proceedings of the Royal Society A, doi:10.1098/rspa.2010.0079, 2010.

Ponomarenko, P. V., Fraser, B. J., Menk, F. W., Ables, S. T., and Morris, R. J.: Cusp-latitude Pc3 spectra: band-limited and power-law components, Ann. Geophys., 20, 1539-1551, doi:10.5194/angeo-20-1539-2002, 2002.

Posch, J. L., Engebretson, M. J., Weatherwax, A. T., Detrick, D. L., Hughes, W. J., and Maclennan, C. G.: Characteristics of broadband ULF magnetic pulsations at conjugate cusp latitude stations, J. Geophys. Res., 104, 311-331, 1999.

Posch, J. L., Engebretson, M. J., Pilipenko, V. A., Hughes, W. J., Russell, C. T., and Lanzerotti, L. J.: Characterizing the longperiod ULF response to magnetic storms, J. Geophys. Res., 108, 1029, doi:10.1029/2002JA009386, 2003.

Potemra, T. A.: Alfven waves and Birkeland currents, Physica Scripta, T60, 107-112, 1995.

Prikryl, P., MacDougall, J. W., Grant, I. F., Steele, D. P., Sofko, G. J., and Greenwald, R. A.: Observations of polar patches generated by solar wind Alfvén wave coupling to the dayside magnetosphere, Ann. Geophys., 17, 463-489, doi:10.1007/s00585999-0463-0, 1999.

Richmond, A. D. and Kamide, Y.: Mapping electrodynamic fea- 
tures of the high-latitude ionosphere from localized observations: Technique, J. Geophys. Res., 93, 5741-5759, 1988.

Samson, J. C.: Three-dimensional polarization characteristics of high-latitude Pc5 geomagnetic micropulsations, J. Geophys. Res., 77, 6145-6160, 1972.

Surkan, A. J. and Lanzerotti, L. J.: ULF Geomagnetic Power near $\mathrm{L}=4$, 3. Statistical Study of Power Spectra in Conjugate Areas during December Solstice, J. Geophys. Res., 79(16), 2403-2412, doi:10.1029/JA079i016p02403, 1974.

Szuberla, C. A. L., Olson, J. V., Engebretson, M. J., McHarg, M. G., and Hughes, W. J.: Spatiotemporal characteristics of cusp latitude spectra, J. Geophys. Res., 105, 7695-7706, 2000.

Takahashi, K. and Anderson, B. J.: Distribution of ULF energy $(f<80 \mathrm{mHz})$ in the inner magnetosphere: A statistical analysis of AMPTE CCE magnetic field data, J. Geophys. Res., 97, 10751-10773, 1992.

Weimer, D.: Maps of ionospheric field-aligned currents as a function of the interplanetary magnetic field derived from Dynamics Explorer 2 data, J. Geophys. Res., 106, 12889-12902, 2001.

Yagova, N., Lanzerotti, L., Villante, U., Pilipenko, V., Lepidi, S., Francia, P., Papitashvili, V., and Rodger, A.: Magnetic activity in the ULF Pc5-6 band at very high latitudes in Antarctica, J. Geophys. Res., 107, 1195, doi:10.1029/2001JA900143, 2002.
Yagova, N. V., Pilipenko, V. A., Lanzerotti, L. J., Engebretson, M. J., Rodger, A. S., Lepidi, S., and Papitashvili, V. O.: Two-dimensional structure of long-period pulsations at polar latitudes in Antarctica, J. Geophys. Res., 109, A03222, doi:10.1029/2003JA010166, 2004.

Yagova, N., Pilipenko, V., Watermann, J., and Yumoto, K.: Control of high latitude geomagnetic fluctuations by interplanetary parameters: the role of suprathermal ions, Ann. Geophys., 25, 1037-1047, doi:10.5194/angeo-25-1037-2007, 2007.

Yagova, N. V., Pilipenko, V. A., Baransky, L. N., Engebretson, M., and Yumoto, K.: Parameters of the geomagnetic noise in the Pc5/Pi3 frequency range for different space weather conditions, Geophysical Research Abstracts, V.10, EGU2008-A11257, SREF-ID:1607/7962/gra/EGU2008-A-11257, 2008.

Zesta, E., Lyons, L., Wang, C.-P., Donovan, E., Frey, H., and Nagai, T.: Poleward boundary intensifications (PBIs): Their two-dimensional structure and associated dynamics in the plasma sheet, J. Geophys. Res., 111, A05201, doi:10.1029/2004JA010640, 2006. 